\title{
Comparison of Rigid Body Motion Trajectory Descriptors for Motion Representation and Recognition
}

\author{
Maxim Vochten ${ }^{1}$, Tinne De Laet ${ }^{2}$, and Joris De Schutter ${ }^{1}$
}

\begin{abstract}
This paper presents an overview and comparison of minimal and complete rigid body motion trajectory descriptors, usable in applications like motion recognition and programming by demonstration. Motion trajectory descriptors are able to deal with potentially unwanted variations acting on the motion trajectory such as changes in the execution time, the motion's starting position, or the viewpoint from which the motion is observed. A suitable rigid body motion trajectory descriptor retains only the trajectory information relevant to the application. This paper compares different trajectory descriptors for rigid body motion and validates their usefulness for dealing with motion variation in a motion recognition experiment. Furthermore, a new type of invariant trajectory descriptor is introduced based on the Frenet-Serret formulas.
\end{abstract}

\section{INTRODUCTION}

The motion trajectory of an object is used in various robotic applications such as motion recognition in humanrobot interaction [1], motion analysis and perception [2], and learning object manipulation tasks in robot programming by human demonstration [3]. The motion trajectory serves as an important tool for representing motion in addition to information about the object's properties, the environment in which the motion happens, or the pose of the manipulator holding the object.

The motion trajectory of a rigid body, such as the end effector of a robot, a human body part, or a human-manipulated object is often described by the trajectory of a single point on the rigid body expressed in a specific reference frame as a function of time [1], [4]. However, this trajectory representation has several shortcomings, such as not taking the rotation of the body into account. Including rotation next to translation is necessary for providing a complete description of the rigid body motion trajectory. The motion trajectory is furthermore influenced by two main types of motion variation. The first type of motion variation is not inherent to the motion itself, but rather to where the motion takes place in space and the choice of references used for expressing the coordinates. This type of motion variation includes:

- Changes in starting position and orientation: The object starts moving from a certain starting position and orientation with respect to the reference frame. Changing this starting location also changes the coordinates of the motion trajectory.

\footnotetext{
${ }^{1} \mathrm{M}$. Vochten and J. De Schutter are with the Department of Mechanical Engineering, KU Leuven, Belgium. maxim.vochten@kuleuven.be and joris. deschutter@kuleuven. be

${ }^{2} \mathrm{~T}$. De Laet is with the Faculty of Engineering Science, KU Leuven, Belgium. tinne.delaet@kuleuven.be
}

- Changes in reference frame: The trajectory coordinates are expressed in a certain reference frame, for example the coordinate frame attached to an observing camera. After moving the camera to a new position or orientation, the observed trajectory coordinates of the same motion will be different.

- Changes in reference point on the body: The translation of a rigid body is usually characterized by the trajectory of a reference point on the body. Choosing a different reference point results in a different trajectory.

The second type of motion variation is due to changes inherent to the execution of the motion itself (the spatial and temporal motion parameters). This type of motion variation includes:

- Changes in execution time and velocity profile: performing the motion faster or slower in time and changing the velocity along the trajectory.

- Changes in amplitude: executing the motion larger or smaller in space.

Choosing appropriate motion trajectory descriptors in applications is important, since it allows to eliminate unwanted dependencies on these changes. For example, in motion recognition the search space for matching trajectories during the classification phase is vastly reduced by using a trajectory descriptor that eliminates unwanted variation, resulting in an improved classification rate. For programming by demonstration, appropriate descriptors capture only the essence of the demonstrated motion trajectories in compact motion primitives, usable for reconstructing and generalizing motion trajectories to a large variety of different contexts.

\section{A. Related work on trajectory descriptors}

Viewpoint-invariant trajectory descriptors have mostly been proposed for point curves, hereby not taking into account rigid body rotation. In the case of a single camera setup, these 3D point curves are projected on a 2D image plane so that descriptors need to be found that remain invariant under projective or affine transformations. An overview of different algebraic, geometric, and differential curve descriptors for describing object shape contours in a viewinvariant way is provided in [5], intended to be used in the context of object recognition. In [6] the curvature scale space and centroid distance are proposed for recognizing activities from video, invariant of viewpoint. The spatio-temporal curvature was used in [7] to segment trajectories in several distinct actions to be recognized. Space-time invariants for a set of individual points on a moving body are proposed in 


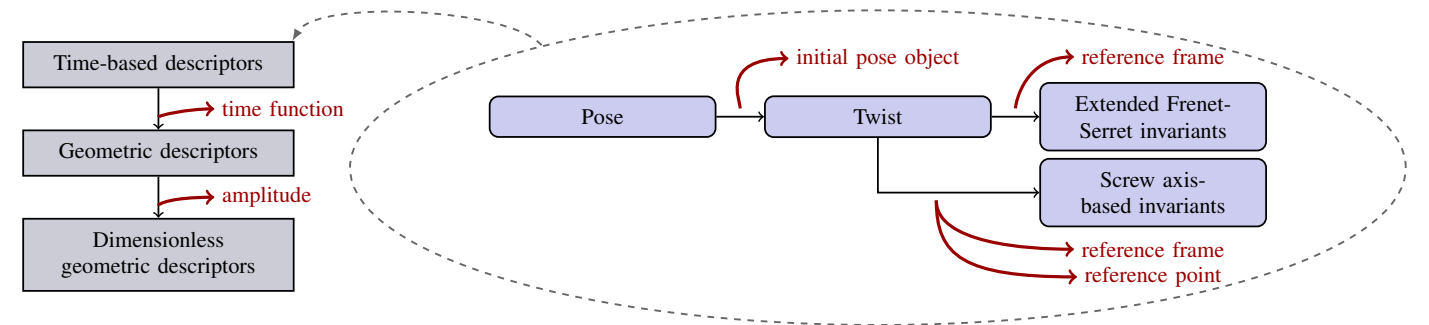

Fig. 1: Overview of rigid body motion trajectory descriptors. On the right, the calculation of time-based trajectory descriptors starting from the rigid body pose is shown. These time-based descriptors are subsequently transformed to geometric and dimensionless geometric descriptors on the left. The red arrows indicate parameters or dependencies influencing the motion trajectory that are extracted during the transformations.

[8]. These are geometric invariants based on the cross ratio of determinants of image coordinates. Comparison between different rigid body motions is possible if the correspondence between the sets of points is known.

If the 3D motion is completely measured by a stereo camera system or an RGB-D camera, the descriptors need to remain invariant only under Euclidean transformations. The differential descriptors in [9] use the local curvature and torsion of a space curve for a coordinate-free description of a point trajectory. These functions are view-invariant, since they only depend on the shape of the curve. Invariance with respect to time was obtained by expressing these descriptors as a function of arc length instead of time. The possibility to extend these local descriptors with global geometric invariants like the centroid distance function for increased robustness is shown in [2]. The differential invariants proposed in [10] describe the complete 3D translation and rotation of a rigid body. This trajectory descriptor, based on the concept of the instantaneous screw axis, is view-invariant and invariant with respect to the choice of reference point used to express the translation of the body. Invariance to changes in motion execution style is obtained by deriving geometric and dimensionless geometric invariants from the time-based invariants.

\section{B. Paper overview}

The structure of the remainder of the paper is as follows. Section III provides an overview of time-based rigid body motion trajectory descriptors for handling variations caused by changes of the starting position and orientation of the object, the choice of reference frame, and reference point. A new type of trajectory descriptor is introduced based on the Frenet-Serret invariants. Section III discusses geometric and dimensionless geometric descriptors for dealing with variation caused by changes in execution style. Figure 11 serves as a guiding figure for the discussion in Section II and III Section IV] illustrates how descriptors deal with motion variation in a motion recognition experiment. Finally, Section V gives conclusions and recommendations for choosing the most appropriate rigid body motion trajectory descriptor.

\section{TIME-BASED TRAJECTORY DESCRIPTORS}

This section provides an overview of rigid body motion trajectory descriptors expressed as a function of time, the time-based trajectory descriptors. These descriptors allow to deal with variations of the rigid body motion trajectory due to changes in starting pose of the object, the reference frame in which the motion is observed, and the reference point for expressing the translation. Starting point of the overview is the rigid body motion trajectory itself. Afterwards, we specify trajectory descriptors to address the variations covered above. For each subsequently introduced descriptor, an additional level of invariance is gained on top of the invariant properties of the previous descriptor.

The discussed rigid body motion trajectory descriptors are minimal. Since rigid body motion is fully characterized by six degrees of freedom (three for translation and three for rotation), each descriptor therefore consists of six functions. The descriptors are also complete, meaning that the original trajectory can be reconstructed from the descriptor and that new trajectories may be generated given some initial parameters. These initial parameters are also briefly stated for each descriptor.

A working example motion "pouring a jug", shown in Figure 2, illustrates the descriptors to be discussed. For this example motion a rigid body trajectory description is needed since the orientation of the jug along the trajectory is as important as the translation.

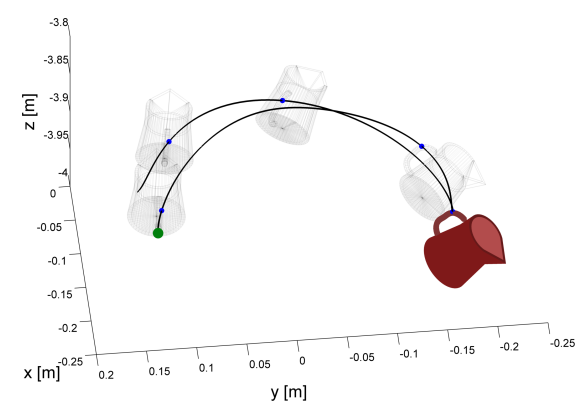

Fig. 2: Example rigid body motion: "pouring a jug". A point on the handle (blue dot) is chosen as the reference point for expressing the translation of the body. The green dot indicates the motion start. 

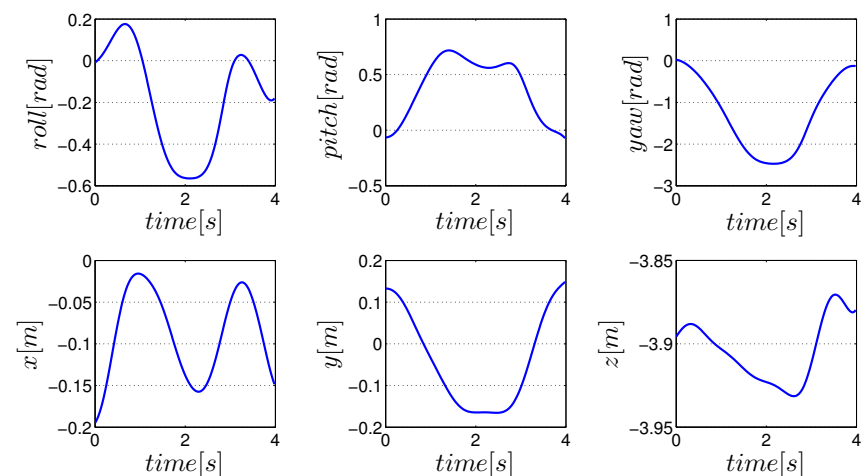

Fig. 3: Pose coordinates of the example motion. The orientation with respect to the reference frame is expressed with roll, pitch, and yaw angles. The translation of the chosen reference point is given by $x, y$, and $z$.

\section{A. Rigid body motion trajectory}

The motion trajectory of a rigid body in three-dimensional space is commonly represented by the pose. The pose consists of two parts: the orientation of the body and the position of a reference point on the body, both expressed with respect to a reference frame. Multiple options exist for expressing the orientation part of the pose [11]. Figure 3 shows the pose coordinates of the example motion "pouring a jug" using roll, pitch, and yaw angles for the orientation and choosing a point on the handle as the reference point for translation.

\section{B. Invariance to changes in starting pose}

The pose coordinates depend on the starting pose (starting position and orientation) of the object. However, this means that after moving the object to a different location in space, different pose coordinates are found for the same motion.

Invariance to the starting pose is realized by using the definition of twist instead. Twist is the generalized velocity for rigid body motion and consists of the translational velocity $\mathbf{v}$ of a reference point on the rigid body together with the rotational velocity $\boldsymbol{\omega}$ of the rigid body with respect to the reference frame. Figure 4 shows the twist for the working example.

Reconstructing the original motion trajectory from the twist requires only the initial pose of the object with respect to the reference frame [12].

\section{Invariance to changes in viewpoint}

The pose and twist coordinates depend on the choice of reference frame in which the translation and rotation are expressed. The reference frame corresponds for example to the coordinate frame attached to a 3D camera observing the motion. However, when the viewpoint of the camera is changed to a different position and/or orientation, the motion trajectory represented by the pose and twist coordinates will be different for the same motion. Figure 5 illustrates the effect of changing viewpoints for the working example. Equivalently, if the camera stays in the same viewpoint, but
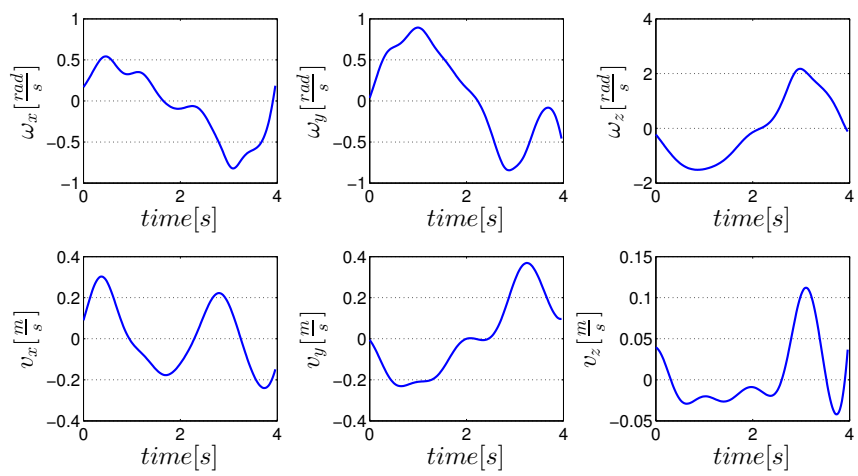

Fig. 4: Twist of the example motion consisting of the rotational velocity $\boldsymbol{\omega}$ of the body and translational velocity $\mathbf{v}$ of the chosen reference point.

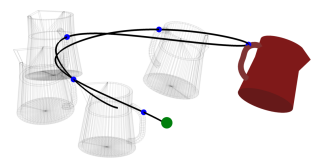

(a)

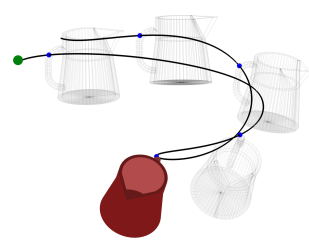

(b)
Fig. 5: Two observations of the same motion from different viewpoints. Though the shape of the motion is geometrically the same, the trajectory looks different in the reference frame of the observer.

the motion is performed in a different direction, the pose and twist are also changed.

Invariance with respect to the reference frame is realized by using a coordinate-free trajectory description, describing the trajectory in terms of the local differential geometry of the curve. For a point curve, this coordinate-free viewinvariant descriptor is given by the Frenet-Serret invariants [13]:

$v_{1}= \pm\|\mathbf{v}\|, v_{2}= \pm \frac{\|\mathbf{v} \times \dot{\mathbf{v}}\|}{\|\mathbf{v}\|^{2}}, v_{3}= \pm \frac{\|(\mathbf{v} \times \dot{\mathbf{v}}) \times(\mathbf{v} \times \ddot{\mathbf{v}})\|}{\|\mathbf{v} \times \dot{\mathbf{v}}\|^{2}}$

The descriptor components $v_{1}, v_{2}$ and $v_{3}$ can be interpreted in a moving Frenet-Serret frame attached to the point curve. While $v_{1}$ corresponds to the motion of the point along one of the axes of the Frenet-Serret frame, $v_{2}$ and $v_{3}$ correspond to the changing orientation of the Frenet-Serret frame itself, resulting in a change of direction in which the point is traveling. The expressions for $v_{2}$ and $v_{3}$ are closely related to the local curvature $\kappa$ and torsion $\tau$ of a point curve:

$$
v_{2}= \pm \kappa\|\mathbf{v}\|, \quad v_{3}= \pm \tau\|\mathbf{v}\|
$$

We extend the Frenet-Serret invariants to rigid body motions by applying the Frenet-Serret formulas to the rotational velocity vector:

$\omega_{1}= \pm\|\boldsymbol{\omega}\|, \omega_{2}= \pm \frac{\|\boldsymbol{\omega} \times \dot{\boldsymbol{\omega}}\|}{\|\boldsymbol{\omega}\|^{2}}, \omega_{3}= \pm \frac{\|(\boldsymbol{\omega} \times \dot{\boldsymbol{\omega}}) \times(\boldsymbol{\omega} \times \ddot{\boldsymbol{\omega}})\|}{\|\boldsymbol{\omega} \times \dot{\boldsymbol{\omega}}\|^{2}}$ 

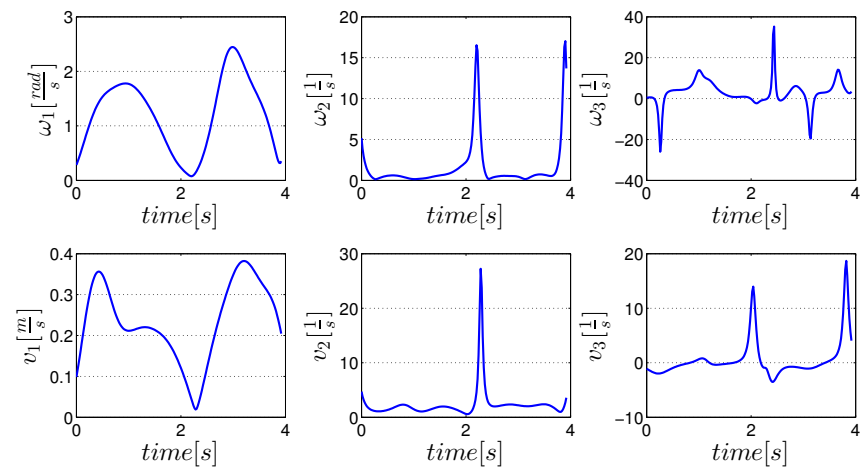

Fig. 6: Extended Frenet-Serret invariants of the example motion.

If the choice of reference point is fixed, we can combine the translational (1) and rotational Frenet-Serret invariants (3) in one descriptor. We denote the resulting new descriptor as the extended Frenet-Serret invariants. (Part of this descriptor $\left(\omega_{2}\right)$ has already been used in [14] to recognize rigid body motions.) Note that if the rigid body performs a pure translation, the extended Frenet-Serret invariants are again reduced to the basic Frenet-Serret invariants of (1). Figure 6 shows the extended Frenet-Serret invariants for the working example.

Reconstructing the original motion trajectory from the extended Frenet-Serret invariants requires initial Frenet-Serret frames for the translational and rotational velocity, indicating the initial direction of translation and rotation with respect to the reference frame. The initial pose of the object is also needed. In [15], a procedure is given to reconstruct a point curve from the curvature and torsion profiles.

\section{Invariance to changes in reference point}

The pose, twist, and extended Frenet-Serret descriptors depend on the choice of reference point to describe the translation. However, in some cases the position of the reference point is uncertain or unknown, for example due to occlusion during a part of the motion. For a different choice of reference point, the motion trajectory descriptors above will be different for the same motion.

Invariance with respect to the reference point is realized using the concept of screw axis. The screw axis is defined by Chasles's theorem [11], which states that a general rigid body motion is always describable by a screw displacement, consisting of a rotation around an axis and a translation along this axis (the screw axis). The translation along the screw axis is independent of the choice of reference point. The same theorem holds at the velocity level. The screw axis is in that case called the Instantaneous Screw Axis (ISA). Figure 7 visualizes the ISA at a certain time step of the motion.

The screw axis-based invariants describe rigid body motion trajectories using the ISA and are presented in [10]. These invariants are similar to the extended Frenet-Serret invariants: the three components describing rotation $\left(\omega_{1}\right.$, $\omega_{2}$, and $\omega_{3}$ ) from (3) are exactly the same, but now the translational velocity $v_{1}$ is defined along the ISA, not with

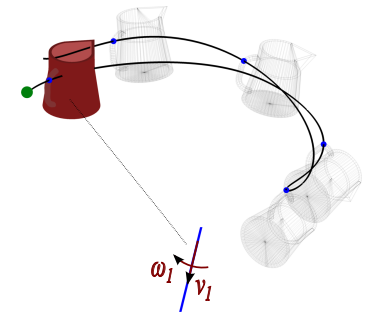

Fig. 7: The Instantaneous Screw Axis (ISA) corresponding to the rigid body motion at a certain time step. The motion is characterized by the rotational velocity $\omega_{1}$ and translational velocity $v_{1}$ along the ISA (blue line).
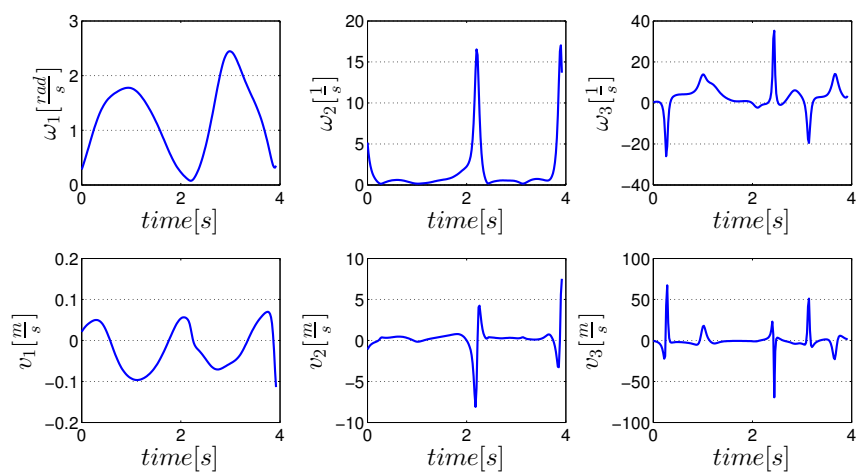

Fig. 8: Screw axis-based invariants of the example motion.

respect to a reference point on the body. The last two components $v_{2}$ and $v_{3}$ correspond to the translation of the moving ISA frame in which all the descriptor components are expressed, similar to the Frenet-Serret frame for the FrenetSerret invariants. The resulting descriptor is invariant with respect to viewpoint changes and the choice of reference point for the translational velocity. Figure 8 shows the screw axis-based invariants for the working example.

Reconstructing the original motion trajectory from the screw axis-based invariants requires an initial ISA frame and the initial pose of the object with respect to the reference frame. The reconstruction procedure is specified in [10].

\section{E. Overview and discussion}

Table I] summarizes all the discussed time-based rigid body motion trajectory descriptors along with their respective invariant properties. It is important to realize that with increasing levels of descriptor invariance, a higher sensitivity to noise is obtained. For example, the extended FrenetSerret invariants are calculated using higher order derivatives that are more susceptible to noise. The same holds for the screw axis-based invariants by relating the translation to the motion of the screw axis. Therefore we recommend to use descriptors only up to the level of invariance required by the application. We also note that the descriptors proposed here are minimal, meaning they can always be complemented by extra contextual information or other local or global descriptors such as the centroid distance function in [6]. 
TABLE I: Overview of time-based rigid body motion trajectory descriptors with their respective invariant properties.

\begin{tabular}{|c|c|c|c|c|}
\hline \multirow[b]{2}{*}{ time-based trajectory descriptor } & \multirow[b]{2}{*}{ consists of } & \multicolumn{3}{|c|}{ invariant with respect to } \\
\hline & & starting pose & viewpoint & choice reference point \\
\hline pose & position reference point and orientation body & - & - & - \\
\hline twist & translational and rotational velocity & $\checkmark$ & - & - \\
\hline extended Frenet-Serret invariants & magnitude velocity, curvature, and torsion & $\checkmark$ & $\checkmark$ & - \\
\hline screw axis-based invariants & velocity along ISA and motion ISA & $\checkmark$ & $\checkmark$ & $\checkmark$ \\
\hline
\end{tabular}

TABLE II: Overview of time-based, geometric, and dimensionless geometric rigid body motion trajectory descriptors with their respective invariant properties.

\begin{tabular}{|c|c|c|c|c|}
\hline \multirow[b]{2}{*}{ trajectory descriptor } & \multirow[b]{2}{*}{ parameterization } & \multicolumn{3}{|c|}{ invariant with respect to } \\
\hline & & time scale & velocity profile & linear/angular scale \\
\hline time-based descriptor & time & - & - & - \\
\hline geometric descriptor & degree of advancement & $\checkmark$ & $\checkmark$ & - \\
\hline dimensionless geometric descriptor & dimensionless degree of advancement & $\checkmark$ & $\checkmark$ & $\checkmark$ \\
\hline
\end{tabular}

\section{GEOMETRIC AND DIMENSIONLESS GEOMETRIC TRAJECTORY DESCRIPTORS}

The descriptors discussed up till now are considered to be a function of time (the time-based descriptors). The motion trajectory is however also influenced by the execution style of the motion, which changes spatial and temporal parameters inherent to the motion such as the total execution time (time scale), the velocity along the trajectory (velocity profile) and the magnitude of the motion (linear/angular scale). Changes to these parameters still affect the time-based descriptors.

This section follows the procedure in [10] to make each time-based descriptor invariant with respect to time, resulting in geometric descriptors. Afterwards, each descriptor is also made invariant with respect to the linear/angular scale resulting in dimensionless geometric descriptors. Table II provides an overview of time-based, geometric and dimensionless geometric descriptors with their respective invariant properties.

\section{A. Invariance to changes in time scale and velocity profile}

The dependence on time of the time-based descriptors is eliminated by expressing the descriptor not as a function of time, but as a function of a degree of advancement. The degree of advancement $\xi(t)$ is a scalar indicating the progress along the trajectory. For a point curve, the arc length is a natural choice for the degree of advancement. For rigid body motion such a natural choice does not exist. Instead we follow the approach of [10] and linearly combine the progression in translation and rotation by defining the time derivative of $\xi(t)$, the rate of advancement $\dot{\xi}(t)$, as follows:

$$
\dot{\xi}(t)=w \frac{\|\boldsymbol{\omega}\|}{\Theta_{s}}+(1-w) \frac{\|\mathbf{v}\|}{L_{s}},
$$

where $\Theta_{s}$ and $L_{s}$ are user-defined scaling factors and $w$ is a chosen dimensionless weight $(0 \leq w \leq 1)$. (For the screw axis-based invariants, $\mathbf{v}$ refers to the velocity along the screw axis.)

Each time-based descriptor component $i_{k}$ (one of the six functions the descriptor comprises) is subsequently reparametrized to its geometric counterpart $I_{k}$ by dividing it with the rate of advancement $\dot{\xi}(t)$, inverting $\xi(t)$, and substituting $t$ by $\xi$ :

$$
I_{k}(\xi) \leftarrow \frac{i_{k}(t(\xi))}{\dot{\xi}(t(\xi))} \text { for } k=1 \ldots 6 .
$$

After this re-parameterization, invariance with respect to the execution time and velocity along the trajectory is obtained, yielding the geometric descriptor.

Reconstructing the original time-based descriptor from the geometric descriptor requires applying the inverse transformation given the degree of advancement and time scale.

\section{B. Invariance to changes in linear/angular scale}

To also eliminate the dependence on linear and angular scale, the degree of advancement is first scaled to 1 by choosing appropriate scaling factors in the rate of advancement (4):

$$
\Theta=\int_{t_{0}}^{t_{f}}\|\boldsymbol{\omega}\| d t, \quad L=\int_{t_{0}}^{t_{f}}\|\mathbf{v}\| d t
$$

where $t_{0}$ is the start time of the motion and $t_{f}$ the end time. Each time-based descriptor component $i_{k}$ is again re-parameterized to their geometric counterpart (5). The descriptor components that are still expressed in radians or meters, are then respectively divided by $\Theta$ and $L$, yielding the dimensionless geometric descriptor 1 .

Reconstructing the original time-based descriptor from the dimensionless geometric descriptor requires applying the inverse transformation given the degree of advancement, time scale, and the linear and angular scale.

\section{MOTION RECOGNITION EXPERIMENT}

The usefulness of invariant trajectory descriptors for eliminating motion variation is illustrated with a motion recognition experiment in which the motion of a human-manipulated object is recognized by means of the rigid body motion trajectory. The focus of the experiments is not to reach the

\footnotetext{
${ }^{1}$ In [10], all components of the screw axis-based invariants were divided by either $\Theta$ or $L$. This needs to be corrected since some of the components (specifically $\omega_{2}$ and $\omega_{3}$ ) were already scale-invariant from the start.
} 


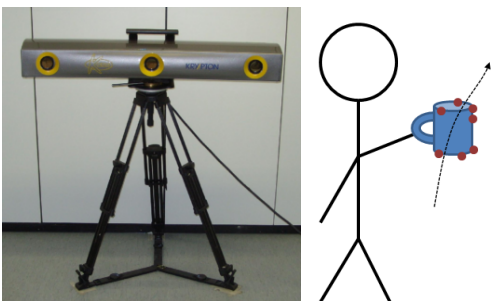

(a)

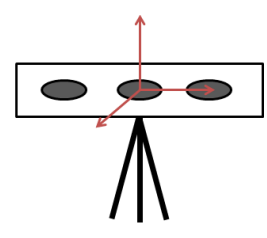

(b)
Fig. 9: Krypton K600 camera system measures the position of LED markers attached to a moving object.

highest possible classification rate for this particular case, but rather to compare the effect of different trajectory descriptors using the same classification algorithm.

The pipeline for recognizing motions starting from the measurements was extensively explained in [16]. The three main differences here are that a new type of trajectory descriptor is considered (the extended Frenet-Serret invariants), that not only the screw axis-based invariants are made dimensionless, and that the effect of variations in the choice of reference point are studied.

\section{A. Experimental setup}

The motion trajectory of a human-manipulated rigid object (a coffee mug) is measured using the Krypton K600 camera system from NIKON Metrology (Figure 9). The Krypton system tracks several LED markers attached to the object and determines the 3D point trajectory of each LED with respect to the reference frame of the camera system. At least three visible LED markers are needed at any given time to describe the translation and orientation of the rigid body.

\section{B. Measurement dataset}

Ten different classes of everyday motions were performed with the object such as shaking, wiping a table, and pouring a drink. To test the invariant properties of the trajectory descriptors, we introduced different kinds of motion variation during the measurements. Measurements were taken from three different viewpoints of the camera (Figure 10) to obtain a change in reference frame. In each viewpoint four different execution styles of the motion were performed by the person handling the object. The four execution styles are: "normal" (the reference execution style), "slower" (smaller time scale), "larger" (larger linear scale), and "faster/slower" (change in velocity profile along the trajectory). Each combination of viewpoint and execution style was executed ten times for each of the ten motion classes yielding a total dataset of 1200 measured motions.

\section{Processing pipeline}

The processing pipeline shown in Figure 11 illustrates how the measured LED marker trajectories are first preprocessed, then transformed to the specified descriptor, and finally classified using model trajectory descriptors for each motion class. The different steps in the pipeline are explained below.
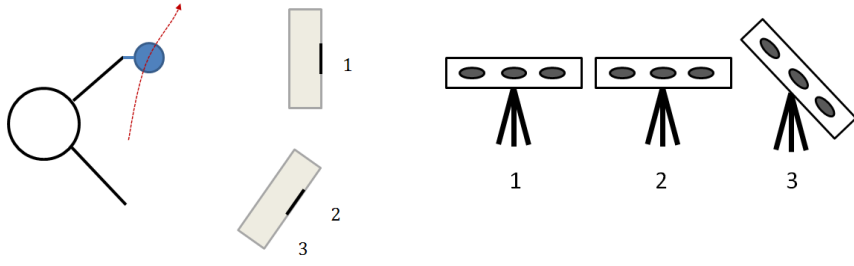

Fig. 10: Representation of how the motions are measured by the camera in three different viewpoints.

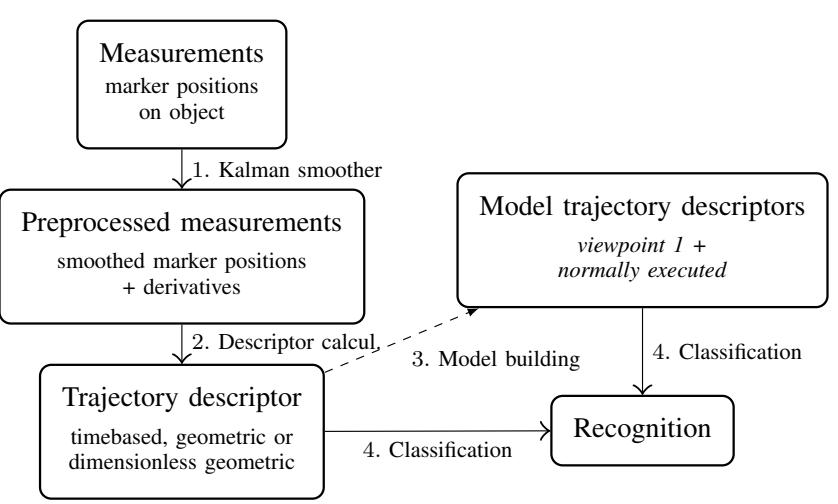

Fig. 11: Processing pipeline from measured LED marker trajectories to classification of the corresponding trajectory descriptor using model trajectory descriptors of all the different motion classes.

1) Kalman smoother: The Kalman smoother serves two purposes: smoothing of the measured LED marker trajectories to reduce noise and calculation of the time derivatives (velocity, acceleration, and jerk) of the marker trajectories in a numerically stable way. The linear Kalman smoother uses a constant derivative jerk model as its process model.

2) Descriptor calculation: Results from the Kalman smoother are first used to calculate the twist and its derivatives. The reference point for the translation is taken as the average position $\mathbf{p}_{c}$ of all the LED markers. The twist components $\boldsymbol{\omega}$ and $\mathbf{v}$ are calculated by solving an overdetermined system of equations using the positions $\mathbf{p}$ and velocities $\dot{\mathbf{p}}$ of the $m$ visible markers:

$$
\dot{\mathbf{p}}_{i}=\mathbf{v}+\boldsymbol{\omega} \times\left(\mathbf{p}_{i}-\mathbf{p}_{c}\right), \quad i=1, \ldots, m .
$$

The twist derivatives are likewise found by solving the system of derived equations (7). Every time-based, geometric, and dimensionless descriptor can now be calculated using the twist and its derivatives (Section III and III).

3) Model building: To validate that the descriptors perform well under motion variation, we build model trajectory descriptors for each motion class using a set of motions (model set) recorded in the first viewpoint and normally executed. The motions from all the other execution styles and viewpoints are classified using these models.

The model building itself is based on the Dynamic Time Warping (DTW) algorithm [17]. The model trajectory is constructed as the DTW distance average motion trajectory of the trajectories in the model set. Figure 12 shows an 


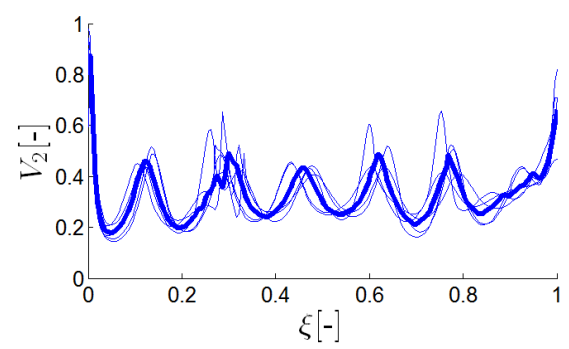

Fig. 12: Model (thick line) of the $V_{2}$ component of the dimensionless extended Frenet-Serret invariants of the "table wiping" motion, constructed as the DTW distance average of five trials in the model set (thin lines).

example. An exponential likelihood function $p\left(D T W_{i j} \mid c_{j}\right)$ is calculated using the DTW distance between every trajectory $i$ and the model trajectory $j$ of the motion. This function is used in the classification step and represents the probability of a motion trajectory being at a certain DTW distance from the model trajectory, given that it belongs to this class of motions $c_{j}$.

4) Classification: The trajectory descriptor of the measured motion is classified using the models of the previous step. First, the DTW distance of the considered descriptor $i$ to every model $j$ is determined: $D T W_{i j}$. Then, the likelihood of this DTW distance is calculated using the exponential likelihood functions $p\left(D T W_{i j} \mid c_{j}\right)$ from the model building step. Finally, the motion is classified by selecting the motion class yielding the highest a posteriori likelihood $p\left(D T W_{i j} \mid c_{j}\right) p\left(c_{j}\right)$, given a priori probabilities $p\left(c_{j}\right)$ for each motion class. These are all set equal in this experiment.

\section{Results and discussion}

The classification results of the motion recognition experiments are given in Table III The model trajectory descriptors were built from motions of viewpoint 1 and execution style "normal", explaining why every descriptor scores $100 \%$ in that column. Though a wide variety of rigid body motion trajectory descriptors are available to be discussed, we compare the following interesting ones in our analysis:

1) Twist (dimensionless): The dimensionless twist performs better than the time-based twist since it is able to cope with changes in execution style. Though not invariant with respect to viewpoint, we also see the performance in viewpoint 2 and 3 increase thanks to the gained invariance to time, velocity, and amplitude changes.

2) Extended Frenet-Serret invariants (dimensionless): The extended Frenet-Serret invariants perform better in viewpoints 2 and 3 than the dimensionless twist, as is expected from its view-invariant properties. However, the results also indicate a loss in viewpoint 1 with respect to the dimensionless twist. This is due to the fact that the descriptor loses some of its selectiveness with respect to the twist. The twist is for example able to differentiate between the three components of the translational velocity vector while the extended Frenet-Serret invariants aggregate this vector into

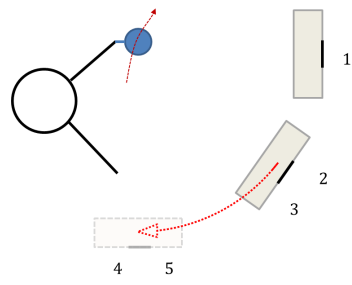

(a)

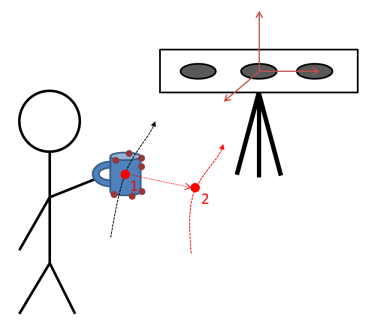

(b)
Fig. 13: (a) Artificially moving the viewpoints further away from the model viewpoint. (b) Choosing a different reference point from the reference point used in the models.

TABLE IV: Percentages of correctly classified motion trajectories for all recorded viewpoints, before and after changing the choice of reference point for the translation. The model trajectories remain unchanged (original reference point).

\begin{tabular}{ll|c} 
Viewpoint 1, 2 \& 3 & average [\%] \\
\hline twist & -time-based & 61 \\
twist & -dimensionless & 83 \\
extended Frenet-Serret invariants & -dimensionless & 83 \\
screw axis-based invariants & -dimensionless & 73 \\
Viewpoint 1, 2 \& 3 (changed reference point) & average [\%] \\
\hline twist & -time-based & 51 \\
twist & -dimensionless & 71 \\
extended Frenet-Serret invariants & -dimensionless & 63 \\
screw axis-based invariants & -dimensionless & 73
\end{tabular}

one scalar, the magnitude. Information about the direction of the vector is subsequently lost.

3) Screw axis-based invariants (dimensionless): The screw axis-based invariants score worse than the extended Frenet-Serret invariants, although there is an extra invariance to changes in reference point. This is due to the higher sensitivity to noise, which arises from defining the translational components with respect to the instantaneous screw axis, as opposed to a reference point. Furthermore, in our experiments the location of the reference point (taken as the average of the marker positions) is not changing much between motions, explaining why the extended Frenet-Serret invariants are a more suitable choice here.

To further illustrate the view-invariant property of the last two descriptors, we introduce an extra artificial transformation from viewpoints 2 and 3 to viewpoints 4 and 5, shown in Figure 13a. The results in Table III show that the two viewinvariant descriptors retain their high percentages in the new viewpoints, while the twist descriptors perform worse.

Finally, we illustrate the invariance to changes in choice of reference point by changing the reference point to a different location relative to the markers from the one assumed in the models (average of marker positions), shown in Figure $13 \mathrm{~b}$ The changed trajectory descriptors are classified using the unchanged models. Table IV] shows that only the results for the screw-axis based descriptors remain unchanged, as expected. 
TABLE III: Percentages of correctly classified motion trajectories recorded under different combinations of viewpoint and execution style, described by different kinds of motion trajectory descriptors. The numbers in bold indicate where a high percentage is expected as a result of invariant properties. Model trajectories for each trajectory descriptor are constructed using motions from viewpoint 1 , normally executed.

\begin{tabular}{|c|c|c|c|c|c|c|}
\hline \multirow{2}{*}{ Viewpoint 1} & & \multicolumn{4}{|c|}{ Execution style } & \multirow[b]{2}{*}{ average $[\%]$} \\
\hline & & normal & larger & faster/slower & slower & \\
\hline twist & -time-based & 100 & 94 & 47 & 74 & 76 \\
\hline twist & -dimensionless geometric & 100 & 94 & 92 & 100 & 96 \\
\hline extended Frenet-Serret invariants & -dimensionless geometric & 100 & 81 & 72 & 90 & 84 \\
\hline screw axis-based invariants & -dimensionless geometric & 100 & 79 & 66 & 85 & 80 \\
\hline \multicolumn{2}{|l|}{ Viewpoint $2 \& 3$} & normal & larger & faster/slower & slower & average $[\%]$ \\
\hline twist & -time-based & 86 & 57 & 29 & 41 & 54 \\
\hline twist & -dimensionless geometric & 80 & 76 & 76 & 77 & 77 \\
\hline extended Frenet-Serret invariants & -dimensionless geometric & 93 & 82 & 72 & 82 & 83 \\
\hline screw axis-based invariants & -dimensionless geometric & 86 & 68 & 59 & 65 & 70 \\
\hline \multicolumn{2}{|c|}{ Viewpoint 4 \& 5 (artificially changed viewpoints) } & normal & larger & faster/slower & slower & average $[\%]$ \\
\hline twist & -time-based & 25 & 24 & 14 & 22 & 21 \\
\hline twist & -dimensionless geometric & 31 & 37 & 23 & 30 & 30 \\
\hline extended Frenet-Serret invariants & -dimensionless geometric & 93 & 82 & 72 & 82 & 83 \\
\hline screw axis-based invariants & -dimensionless geometric & 86 & 68 & 59 & 65 & 70 \\
\hline
\end{tabular}

\section{CONCLUSIONS AND RECOMMENDATIONS}

The overview of rigid body motion trajectory descriptors presented in this paper is meant as a guideline for choosing the most appropriate trajectory descriptor in applications like motion recognition and programming by demonstration. The choice of descriptor is based on which variations in the observed motion trajectories need to be eliminated.

We recommend using invariant descriptors only up to the level of invariance required by the application. Since including additional invariant properties increases the sensitivity of the descriptor to noise, the potential effectiveness of the trajectory descriptor would be limited in that application.

\section{SOFTWARE}

MATLAB code for calculating all the rigid body motion trajectory descriptors considered in this paper has been made publicly available to download at [18].

\section{ACKNOWLEDGMENT}

All authors gratefully acknowledge the financial support by the Flemish FWO project G0B6613.

\section{REFERENCES}

[1] S. Bodiroža, G. Doisy, and V. V. Hafner, "Position-invariant, real-time gesture recognition based on dynamic time warping," in Proceedings of the 8th ACM/IEEE International Conference on Human-robot Interaction, Tokyo, Japan, 2013, pp. 87-88.

[2] J. Yang, Y. Li, K. Wang, Y. Wu, G. Alieri, and M. Scalia, "Mixed signature: An invariant descriptor for 3D motion trajectory perception and recognition," Mathematical Problems in Engineering, vol. 2012, 2012.

[3] M. Ferreira, P. Costa, L. Rocha, and A. Moreira, "Stereo-based realtime 6-dof work tool tracking for robot programing by demonstration," The International Journal of Advanced Manufacturing Technology, pp. 1-13, 2014.

[4] S. Calinon, F. Guenter, and A. Billard, "On learning, representing and generalizing a task in a humanoid robot," IEEE Transactions on Systems, Man, and Cybernetics. Part B: Cybernetics, vol. 37, no. 2, pp. 286-298, 2007.
[5] I. Weiss, "Geometric invariants and object recognition," International Journal of Computer Vision, vol. 10, no. 3, pp. 207-231, 1993

[6] F. I. Bashir, A. A. Khokhar, and D. Schonfeld, "View-invariant motion trajectory-based activity classification and recognition," Multimedia Systems, 2006.

[7] C. Rao, A. Yilmaz, and M. Shah, "View-invariant representation and recognition of actions," International Journal of Computer Vision, vol. 50, no. 2, pp. 203-226, 2002.

[8] Y. Piao, K. Hayakawa, and J. Sato, "Space-time invariants for recognizing 3D motions from arbitrary viewpoints under perspective projection," Transactions of the Institute of Electronics, Information and Communication Engineers, vol. E89-D, no. 7, pp. 2268-2274, 2006.

[9] S. Wu and Y. F. Li, "Flexible signature descriptions for adaptive motion trajectory representation, perception and recognition," Pattern Recognition, vol. 42, no. 1, pp. 194-214, January 2009.

[10] J. De Schutter, "Invariant description of rigid body motion trajectories," Transactions of the ASME, Journal of Mechanisms and Robotics, vol. 2, no. 1, pp. 011 004/1-9, 2010.

[11] K. Waldron and J. Schmiedler, "Kinematics," in Handbook of Robotics. Springer-Verlag, Berlin, Heidelberg, 2008, ch. A.1, pp. 9-33.

[12] R. P. Paul, Robot Manipulators: Mathematics, Programming, and Control. Cambridge, MA: MIT Press, 1981.

[13] W. Kühnel, Differential geometry: curves-surfaces-manifolds. Providence, RI: American Mathematical Society, 2006.

[14] M. Saveriano and D. Lee, "Invariant representation for user independent motion recognition," in IEEE International Symposium on Robot and Human Interactive Communication, Gyeongju, Korea (South), August 2013, pp. 650-655.

[15] S. Wu and Y. Li, "Motion trajectory reproduction from generalized signature description," Pattern Recognition, vol. 43, no. 1, pp. $204-$ 221, 2010.

[16] T. Delabie, O. Cigdem, J.F.M. De Schutter, R. Matthysen, T. De Laet, and J. De Schutter, "Invariant representations to reduce the variability in recognition of rigid body motion trajectories," in IEEE International Conference on Systems, Man, and Cybernetics, Oct 2012, pp. 16581663.

[17] H. Sakoe and S. Chiba, "Dynamic programming algorithm optimization for spoken word recognition," IEEE Transactions on Acoustics, Speech, and Signal Processing, vol. 26, no. 1, pp. 43-49, 1978.

[18] M. Vochten, "Rigid Body Motion Trajectory Descriptors," http://www. mech.kuleuven.be/en/pma/research/robotics/research/invariants 2015, last visited February 2015. 